\title{
Innovative methods of correlation and orbit determination for space debris
}

\author{
Farnocchia D. ${ }^{1}$, Tommei G. ${ }^{1}$, Milani A. ${ }^{1}$ and Rossi A. ${ }^{2}$ \\ ${ }^{1}$ Department of Mathematics, University of Pisa, \\ Largo Bruno Pontecorvo 5, 56127 Pisa, Italy \\ e-mail: farnocchia@mail.dm.unipi.it, \{tommei,milani\}@dm.unipi.it \\ ${ }^{2}$ ISTI/CNR, Via Moruzzi 1, 56124 Pisa, Italy \\ e-mail: alessandro.rossi@isti.cnr.it
}

\begin{abstract}
We propose two algorithms to provide a full preliminary orbit of an Earth-orbiting object with a number of observations lower than the classical methods, such as those by Laplace and Gauss. The first one is the Virtual debris algorithm, based upon the admissible region, that is the set of the unknown quantities corresponding to possible orbits for a given observation for objects in Earth orbit (as opposed to both interplanetary orbits and ballistic ones). A similar method has already been successfully used in recent years for the asteroidal case. The second algorithm uses the integrals of the geocentric 2-body motion, which must have the same values at the times of the different observations for a common orbit to exist. We also discuss how to account for the perturbations of the 2-body motion, e.g., the $J_{2}$ effect.
\end{abstract}

Keywords: Space debris, Orbit determination, Admissible region, Keplerian integrals

\section{Introduction}

The near-Earth space, filled by more than 300000 artificial debris particles with diameter larger than $1 \mathrm{~cm}$, can be divided into three main regions: the Low Earth Orbit (LEO), below about $2000 \mathrm{~km}$, the Medium Earth Orbit (MEO), above $2000 \mathrm{~km}$ and below $36000 \mathrm{~km}$, and the Geosynchronous Earth Orbit (GEO) at about $36000 \mathrm{~km}$ of altitude. Currently the orbits of more than 12000 objects larger than about $10 \mathrm{~cm}$ are listed in the so called Two Line Elements (TLE) catalogue. To produce and maintain such a catalogue a large number of optical and radar observations are routinely performed by the United States Space Surveillance Network. Nowadays also Europe has launched its Space Situational Awareness (SSA) initiative aimed to increase the knowledge of the 
circumterrestrial environment. In this context the availability of efficient methods and algorithms for accurate orbit determination is extremely important.

Given two or more sets of observations, the main problem is how to identify which separate sets of data belong to the same physical object (the so-called correlation problem). Thus the orbit determination problem needs to be solved in two stages: first different sets of observations need to be correlated, then an orbit can be determined; this combined procedure is called linkage in the literature [Milani 1999].

In this paper we describe two different linkage methods, for both optical and radar data. By using the attributable vector (Sec. 2) we summarize the information contained in either optical or radar data. In Sec. 3 we describe the admissible region and the Virtual debris algorithm [Tommei et al. 2007] and we propose a general scheme to classify observed objects. Sec. 4 deals with the Keplerian integrals method, first introduced by [Gronchi et al. 2009] for the problem of asteroid orbit determination. Furthermore, the inclusion of the effect due to the non-spherical shape of the Earth is discussed. Finally, in Sec. 5, a sketch of the general procedure for the full process of correlation of different observations is outlined.

The spirit of this paper is to propose algorithms suitable for implementation with affordable computational complexity. Indeed there have been many cases in which theoretically interesting algorithms have been proposed but their practical application has faced computational challenges [Maruskin and Scheeres 2009], [Maruskin et al. 2009] and [Taff and Hall 1977].

\section{Observations and attributables}

Objects in LEO are mostly observed by radar while for MEOs and GEOs optical sensors are used. In both cases, the batches of observations which can be immediately assigned to a single object give us a set of data that can be summarized in an attributable, that is a 4-dimensional vector. To compute a full orbit, formed by 6 parameters, we need to know 2 further quantities.

Thus the question is the identification problem, also called correlation in the debris context: given two attributables at different times, can they belong to the same orbiting object? And if this is the case, can we find an orbit fitting both data sets?

Let $(\rho, \alpha, \delta) \in \mathbb{R}^{+} \times[0,2 \pi) \times(-\pi / 2, \pi / 2)$ be topocentric spherical coordinates for the position of an Earth satellite. The angular coordinates $(\alpha, \delta)$ are defined by a topocentric reference system that can be arbitrarily selected. Usually, in the applications, $\alpha$ is the right ascension and $\delta$ the declination with respect to an equatorial reference system (e.g., J2000). The values of range $\rho$ and range rate $\dot{\rho}$ are not measured.

We shall call optical attributable a vector

$$
\mathcal{A}_{o p t}=(\alpha, \delta, \dot{\alpha}, \dot{\delta}) \in[0,2 \pi) \times(\pi / 2, \pi / 2) \times \mathbb{R}^{2},
$$


representing the angular position and velocity of the body at a time $t$ in the selected reference frame.

Active artificial satellites and space debris can also be observed by radar; however, because of the $1 / \rho^{4}$ dependence of the signal to noise for radar observations, range and range-rate are currently measured only for debris in LEO. When a return signal is acquired, the antenna pointing angles are also available. Given the capability of modern radars to scan very rapidly the entire visible sky, radar can be used to discover all the debris above a minimum size while they are visible from an antenna, or a system of antennas.

When a radar observation is performed we assume that the measured quantities (all with their own uncertainty) are the range, the range rate, and also the antenna pointing direction, that is the debris apparent position on the celestial sphere, expressed by two angular coordinates such as right ascension $\alpha$ and declination $\delta$. The time derivatives of these angular coordinates, $\dot{\alpha}$ and $\dot{\delta}$, are not measured.

We define radar attributable a vector

$$
\mathcal{A}_{\text {rad }}=(\alpha, \delta, \rho, \dot{\rho}) \in[-\pi, \pi) \times(-\pi / 2, \pi / 2) \times \mathbb{R}^{+} \times \mathbb{R},
$$

containing the information from a radar observation, at the receive time $t$.

Given the attributable $\mathcal{A}$, to define an orbit the values of two unknowns quantities (e.g., $\rho$ and $\dot{\rho}$ in the optical case, $\dot{\alpha}$ and $\dot{\delta}$ in the radar case) need to be found at the same instance in time as the attributable. These two quantities, together with $\mathcal{A}$, give us a set of attributable orbital elements

$$
X=[\alpha, \delta, \dot{\alpha}, \dot{\delta}, \rho, \dot{\rho}]
$$

at a time $\bar{t}$, computed from $t$ taking into account the light-time correction: $\bar{t}=$ $t-\rho / c$. Of course the information on the observer station must be available. The Cartesian position and velocity $(\mathbf{r}, \dot{\mathbf{r}})$, in a geocentric frame, can be obtained, given the observer geocentric position $\mathbf{q}$ at time $t$, by using the unit vector $\hat{\boldsymbol{\rho}}=(\cos \alpha \cos \delta, \sin \alpha \cos \delta, \sin \delta)$ in the direction of the observation:

$$
\begin{gathered}
\mathbf{r}=\mathbf{q}+\rho \hat{\boldsymbol{\rho}} \quad, \quad \dot{\mathbf{r}}=\dot{\mathbf{q}}+\dot{\rho} \hat{\boldsymbol{\rho}}+\rho \frac{d \hat{\boldsymbol{\rho}}}{d t} \quad, \quad \frac{d \hat{\boldsymbol{\rho}}}{d t}=\dot{\alpha} \hat{\boldsymbol{\rho}}_{\alpha}+\dot{\delta} \hat{\boldsymbol{\rho}}_{\delta}, \\
\hat{\boldsymbol{\rho}}_{\alpha}=(-\sin \alpha \cos \delta, \cos \alpha \cos \delta, 0), \quad \hat{\boldsymbol{\rho}}_{\delta}=(-\cos \alpha \sin \delta,-\sin \alpha \sin \delta, \cos \delta) .
\end{gathered}
$$

\section{Admissible region theory}

Starting from an attributable, we would like to extract sufficient information from it in order to compute preliminary orbits: we shall use the admissible region tool, as described in [Tommei et al. 2007]. For ease of reading, we recall here the basic steps of the theory.

The admissible region replaces the conventional confidence region as defined in the classical orbit determination procedure. The main requirement is that the geocentric energy of the object is negative, so that the object is a satellite of the Earth. 


\subsection{Optical admissible region}

Given the geocentric position $\mathbf{r}$ of the debris, the geocentric position $\mathbf{q}$ of the observer, and the topocentric position $\boldsymbol{\rho}$ of the debris we have $\mathbf{r}=\boldsymbol{\rho}+\mathbf{q}$. The energy (per unit of mass) is given by

$$
\mathcal{E}(\rho, \dot{\rho})=\frac{1}{2}\|\dot{\mathbf{r}}(\rho, \dot{\rho})\|^{2}-\frac{\mu}{\|\mathbf{r}(\rho)\|},
$$

where $\mu$ is the Earth's gravitational parameter. Then a definition of admissible region such that only satellites of the Earth are allowed includes the condition

$$
\mathcal{E}(\rho, \dot{\rho}) \leq 0
$$

that, by substituting (1) in (2), could be rewritten as

$$
\begin{gathered}
2 \mathcal{E}(\rho, \dot{\rho})=\dot{\rho}^{2}+w_{1} \dot{\rho}+T(\rho)-\frac{2 \mu}{\sqrt{S(\rho)}} \leq 0, \\
T(\rho)=w_{2} \rho^{2}+w_{3} \rho+w_{4} \quad, \quad S(\rho)=\rho^{2}+w_{5} \rho+w_{0}
\end{gathered}
$$

and coefficients $w_{i}$ depending on the attributable [Tommei et al. 2007]:

$$
\begin{array}{lll}
w_{0}=\|\mathbf{q}\|^{2}, & w_{1}=2 \dot{\mathbf{q}} \cdot \hat{\boldsymbol{\rho}}, & w_{2}=\dot{\alpha}^{2} \cos ^{2} \delta+\dot{\delta}^{2}=\eta^{2}, \\
w_{3}=2\left(\dot{\alpha} \dot{\mathbf{q}} \cdot \hat{\boldsymbol{\rho}}_{\alpha}+\dot{\delta} \dot{\mathbf{q}} \cdot \hat{\boldsymbol{\rho}}_{\delta}\right), & w_{4}=\|\dot{\mathbf{q}}\|^{2}, & w_{5}=2 \mathbf{q} \cdot \hat{\boldsymbol{\rho}},
\end{array}
$$

where $\eta$ is the proper motion. In order to obtain real solutions for $\dot{\rho}$ the discriminant of $2 \mathcal{E}$ (polynomial of degree 2 in $\dot{\rho}$ ) must be non-negative:

$$
\Delta=\frac{w_{1}^{2}}{4}-T(\rho)+\frac{2 \mu}{\sqrt{S(\rho)}} \geq 0 .
$$

This observation results in the following condition on $\rho$ :

$$
\frac{2 \mu}{\sqrt{S(\rho)}} \geq Q(\rho)=w_{2} \rho^{2}+w_{3} \rho+\gamma \quad, \quad \gamma=w_{4}-\frac{w_{1}^{2}}{4} .
$$

Condition (5) can be seen as an inequality involving a polynomial $V(\rho)$ of degree 6 :

$$
V(\rho)=Q^{2}(\rho) S(\rho) \leq 4 \mu^{2} .
$$

Studying the polynomial $V(\rho)$ and its roots, as done by [Milani et al. 2004], the conclusion is that the region of $(\rho, \dot{\rho})$ such that condition $(3)$ is satisfied can admit more than one connected component, but it has at most two. In any case, in a large number of numerical experiments with objects in Earth orbit, we have not found examples with two connected components.

The admissible region needs to be compact in order to have the possibility to sample it with a finite number of points, thus a condition defining an inner boundary needs to be added. The choice for the inner boundary depends upon 
the specific orbit determination task: a simple method is to add constraints $\rho_{\min } \leq \rho \leq \rho_{\max }$ allowing, e.g., to focus the search of identifications to one of the three classes LEO, MEO and GEO. Another natural choice for the inner boundary is to take $\rho \geq h_{\text {atm }}$ where $h_{a t m}$ is the thickness of a portion of the Earth atmosphere in which a satellite cannot remain in orbit for a significant time span. As an alternative, it is possible to constrain the semimajor axis to be larger than $R_{\oplus}+h_{a t m}=r_{\text {min }}$, and this leads to the inequality

$$
a(\rho, \dot{\rho})=-\frac{\mu}{2 \mathcal{E}(\rho, \dot{\rho})} \geq r_{\text {min }} \Longleftrightarrow \mathcal{E}(\rho, \dot{\rho}) \geq-\frac{\mu}{2 r_{\min }}=\mathcal{E}_{\text {min }},
$$

which defines another degree six inequality with the same coefficients but for a different constant term. The qualitative structure of the admissible region is shown in Fig. 1.

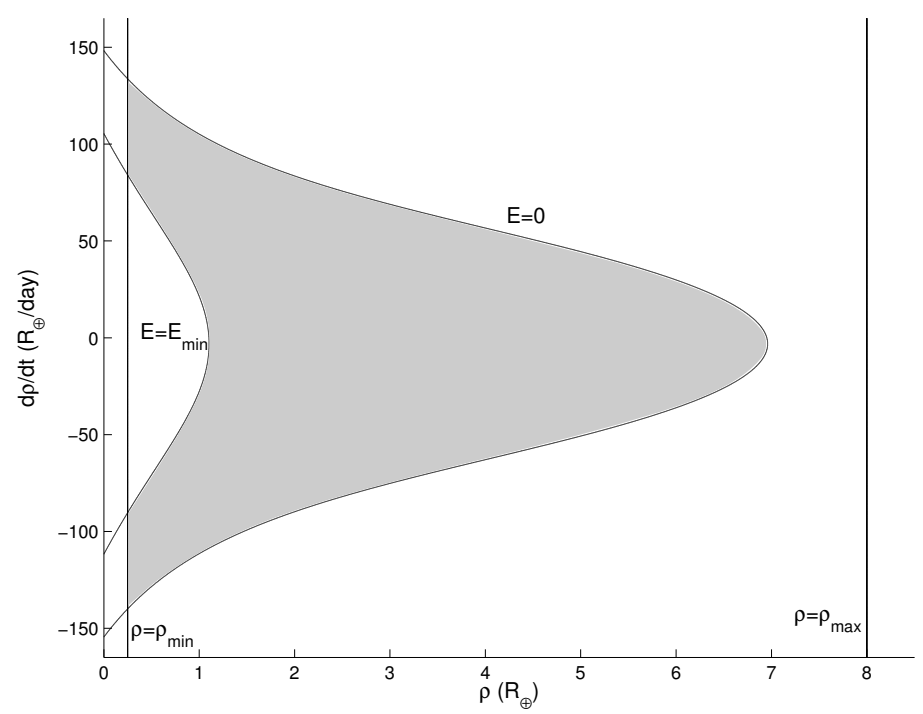

Figure 1: An example of admissible region, optical case, in the $(\rho, \dot{\rho})$ plane. The region (painted in grey) is bounded by two level curves of the energy, $\left(E=E_{\text {min }}\right)$ and $(E=0)$, and by the two conditions on the topocentric distance $\left(\rho=\rho_{\text {min }}\right.$ and $\left.\rho=\rho_{\max }\right)$.

Another possible way to find an inner boundary is to exclude trajectories impacting the Earth in less than one revolution, that is to use an inequality on the perigee $r_{P}$, already proposed in [Maruskin et al. 2009]:

$$
r_{P}=a(1-e) \geq r_{\min } .
$$

Note that this condition naturally implies (6) and $\rho \geq h_{a t m}$. To analytically develop the inequality (7) we need the formulae involving the angular momentum:

$$
\mathbf{c}(\rho, \dot{\rho})=\mathbf{r} \times \dot{\mathbf{r}}=\mathbf{D} \dot{\rho}+\mathbf{E} \rho^{2}+\mathbf{F} \rho+\mathbf{G}
$$




$$
\begin{array}{ll}
\mathbf{D}=\mathbf{q} \times \hat{\boldsymbol{\rho}}, & \mathbf{E}=\hat{\boldsymbol{\rho}} \times\left(\dot{\alpha} \hat{\boldsymbol{\rho}}_{\alpha}+\dot{\delta} \hat{\boldsymbol{\rho}}_{\delta}\right), \\
\mathbf{F}=\mathbf{q} \times\left(\dot{\alpha} \hat{\boldsymbol{\rho}}_{\alpha}+\dot{\delta} \hat{\boldsymbol{\rho}}_{\delta}\right)+\hat{\boldsymbol{\rho}} \times \dot{\mathbf{q}}, & \mathbf{G}=\mathbf{q} \times \dot{\mathbf{q}} .
\end{array}
$$

From the 2-body formulae we have that

$$
a=-\frac{\mu}{2 \mathcal{E}} \quad, \quad a\left(1-e^{2}\right)=\frac{\|\mathbf{c}\|^{2}}{\mu} \Rightarrow e^{2}=1+\frac{2 \mathcal{E}\|\mathbf{c}\|^{2}}{\mu^{2}}
$$

and substituting in (7) we obtain:

$$
\sqrt{1+\frac{2 \mathcal{E}\|\mathbf{c}\|^{2}}{\mu^{2}}} \leq 1+\frac{2 \mathcal{E} r_{\text {min }}}{\mu}
$$

Since the left hand side is $e \geq 0$, we need to impose $1+2 \mathcal{E} r_{\text {min }} / \mu \geq 0$ : this is again $a \geq r_{\min }$. Finally, by squaring (9), we obtain:

$$
\|\mathbf{c}\|^{2} \geq 2 r_{\min }\left(\mu+\mathcal{E} r_{\min }\right) .
$$

Using (4) and (8), the above inequality become algebraic in the variables $(\rho, \dot{\rho})$ :

$$
\left(r_{\text {min }}^{2}-\|\mathbf{D}\|^{2}\right) \dot{\rho}^{2}-P(\rho) \dot{\rho}-U(\rho)+r_{\min }^{2} T(\rho)-\frac{2 r_{\min }^{2} \mu}{\sqrt{S(\rho)}} \leq 0
$$

$$
\begin{aligned}
P(\rho) & =2 \mathbf{D} \cdot \mathbf{E} \rho^{2}+2 \mathbf{D} \cdot \mathbf{F} \rho+2 \mathbf{D} \cdot \mathbf{G}-r_{\min }^{2} w_{1}, \\
U(\rho) & =\|\mathbf{E}\|^{2} \rho^{4}+2 \mathbf{E} \cdot \mathbf{F} \rho^{3}+\left(2 \mathbf{E} \cdot \mathbf{G}+\|\mathbf{F}\|^{2}\right) \rho^{2}+2 \mathbf{F} \cdot \mathbf{G} \rho+\|\mathbf{G}\|^{2}-2 r_{\min } \mu .
\end{aligned}
$$

The coefficient of $\dot{\rho}^{2}$ is positive, thus to obtain real solutions for $\dot{\rho}$ the discriminant of (11) must be non negative:

$$
\Delta_{P}=P^{2}(\rho)+4\left(r_{m i n}^{2}-\|\mathbf{D}\|^{2}\right)\left(U(\rho)-r_{m i n}^{2} T(\rho)+\frac{2 r_{m i n}^{2} \mu}{\sqrt{S(\rho)}}\right) \geq 0 .
$$

This condition is equivalent to the following:

$$
\frac{2 \mu}{\sqrt{S(\rho)}} \geq W(\rho)=-\frac{4\left(r_{\min }^{2}-\|\mathbf{D}\|^{2}\right)\left(U(\rho)-r_{\min }^{2} T(\rho)\right)+P^{2}(\rho)}{4 r_{\min }^{2}\left(r_{\min }^{2}-\|\mathbf{D}\|^{2}\right)} .
$$

Note that the inequality (12) is similar to (5). However, in this case, the function in the right hand side is much more complicated, and there is no easy way to use the condition (7) to explicitly describe the boundary of the admissible region; e.g., we do not have a rigorous bound on the number of connected components. Condition (7) will be used only a posteriori as a filter (Sec. 3.3).

Fig. 2 shows also this inner boundary; note that the boundaries of the regions defined by (6) and by $\rho \geq h_{a t m}$ are also plotted in the figure, but these 


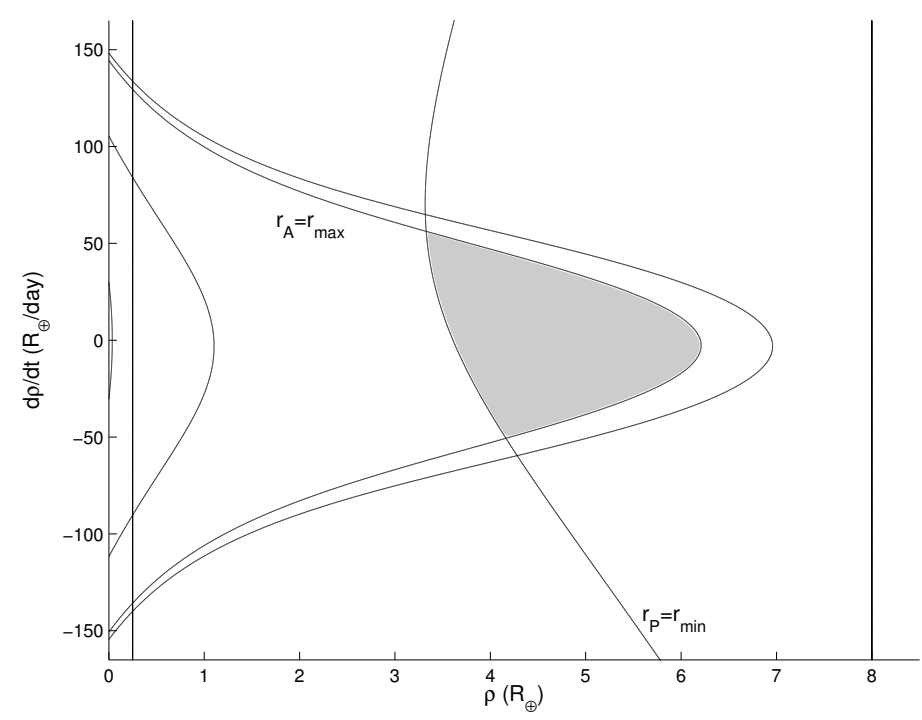

Figure 2: The same example of Fig. 1, with the two further conditions on the pericenter $\left(r_{P} \geq r_{\text {min }}\right)$ and the apocenter $\left(r_{A} \leq r_{\max }\right)$ distances.

constraints are not necessary. We have also plotted an alternative outer boundary constraining the apocenter $r_{A}=a(1+e)$ at some large value $r_{\text {max }}$, described by the following relationships (obtained similarly to the pericenter case):

$$
\left\{\begin{array}{l}
\mathcal{E} \leq-\frac{\mu}{2 r_{\max }} \\
\|\mathbf{c}\|^{2} \geq 2 r_{\max }\left(\mu+\mathcal{E} r_{\text {max }}\right)
\end{array} ;\right.
$$

this outer boundary can be used in the same way, as an a posteriori filter.

\subsection{Radar admissible region}

Given a radar attributable $\mathcal{A}_{\text {rad }}$, we define as radar admissible region for a space debris the set of values of $(\dot{\alpha}, \dot{\delta})$ such that

$$
2 \mathcal{E}(\alpha, \delta)=z_{11} \dot{\alpha}^{2}+z_{22} \dot{\delta}^{2}+2 z_{13} \dot{\alpha}+2 z_{23} \dot{\delta}+z_{33} \leq 0,
$$

where $z_{i j}$ depend on the attributable [Tommei et al. 2007]:

$$
\begin{array}{ll}
z_{11}=\rho^{2} \cos ^{2} \delta, & z_{22}=\rho^{2},
\end{array} \quad z_{13}=\rho \dot{\mathbf{q}} \cdot \hat{\boldsymbol{\rho}}_{\alpha},
$$

The boundary of the admissible region is then given by $\mathcal{E}(\dot{\alpha}, \dot{\delta})=0$ and this equation represents an ellipse with its axes aligned with the coordinate 


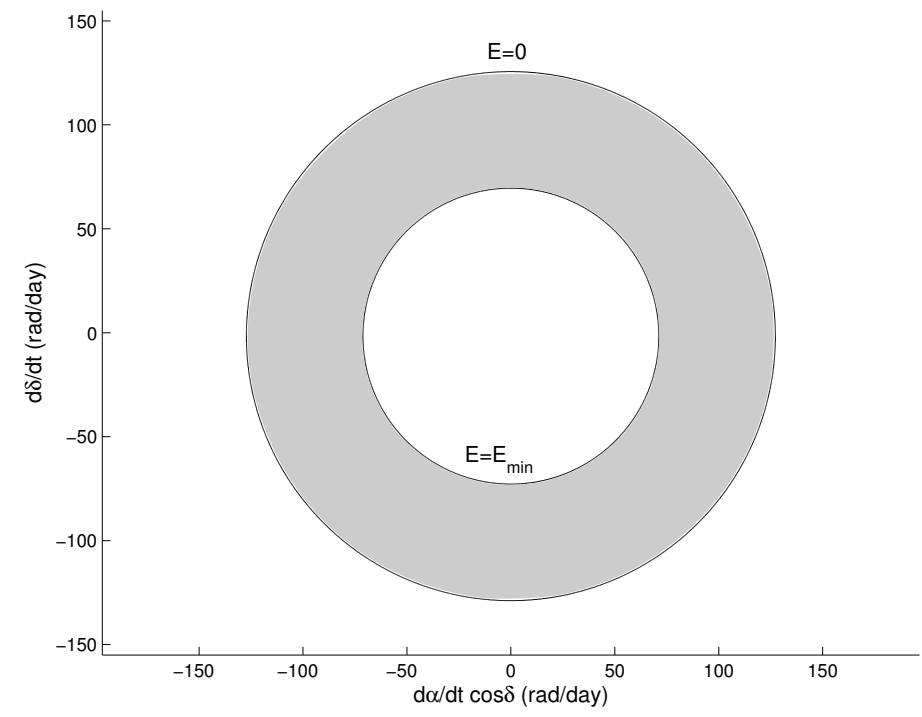

Figure 3: An example of admissible region, radar case, in the $(\dot{\alpha} \cos \delta, \dot{\delta})$ plane. The region (painted in grey) is the circular annulus bounded by the two level curves of the energy $\left(E=E_{\min }\right)$ and $(E=0)$.

axes in the $(\dot{\alpha}, \dot{\delta})$ plane. Actually, in a plane $(\dot{\alpha} \cos \delta, \dot{\delta})$, with the axes rescaled according to the metric of the tangent plane to the celestial sphere, the curves $\mathcal{E}(\dot{\alpha}, \dot{\delta})=$ constant are circles.

The region defined by negative geocentric energy, the inside of a circle, is a compact set, and the problem of defining an inner boundary is much less important than in the optical attributable case. Anyway, it is possible to define an inner boundary by constraining the semimajor axis $a>r_{m i n}$, that is by eq. (6), resulting in a concentric inner circle, thus in an admissible region forming a circular annulus (see Fig. 3).

It is also possible to exclude the ballistic trajectories by imposing the condition (7) in which $\dot{\alpha}, \dot{\delta}$ are to be considered as variables. The angular momentum is given by

$$
\begin{gathered}
\mathbf{c}(\dot{\alpha}, \dot{\delta})=\mathbf{r} \times \dot{\mathbf{r}}=\mathbf{A} \dot{\alpha}+\mathbf{B} \dot{\delta}+\mathbf{C}, \\
\mathbf{A}=\rho \mathbf{r} \times \hat{\boldsymbol{\rho}}_{\alpha}, \quad \mathbf{B}=\rho \mathbf{r} \times \hat{\boldsymbol{\rho}}_{\delta}, \quad \mathbf{C}=\mathbf{r} \times \dot{\mathbf{q}}+\dot{\rho} \mathbf{q} \times \hat{\boldsymbol{\rho}} .
\end{gathered}
$$

By substituting (13) and (14) in (10), the condition on the pericenter is expressed by a polynomial inequality of degree 2 :

$$
l_{11} \dot{\alpha}^{2}+2 l_{12} \dot{\alpha} \dot{\delta}+l_{22} \dot{\delta}^{2}+2 l_{13} \dot{\alpha}+2 l_{23} \dot{\delta}+l_{33} \geq 0,
$$

$$
\begin{array}{lll}
l_{11}=\|\mathbf{A}\|^{2}-r_{m i n}^{2} z_{11}, & l_{12}=\mathbf{A} \cdot \mathbf{B}, & l_{13}=\mathbf{A} \cdot \mathbf{C}-r_{m i n}^{2} z_{13}, \\
l_{22}=\|\mathbf{B}\|^{2}-r_{\text {min }}^{2} z_{22}, & l_{23}=\mathbf{B} \cdot \mathbf{C}-r_{m i n}^{2} z_{23}, & l_{33}=\|\mathbf{C}\|^{2}-r_{m i n}\left(r_{m i n} z_{33}+2 \mu\right) .
\end{array}
$$


Thus the admissible region can be geometrically described as a region bounded by three conics: the first two are concentric circles, the third one can be either an ellipse or a hyperbola (depending on the sign of $l_{11} l_{22}-l_{12}^{2}$ ), with a different center and different symmetry axes. Fig. 4 and 5 show the possible qualitatively different cases.

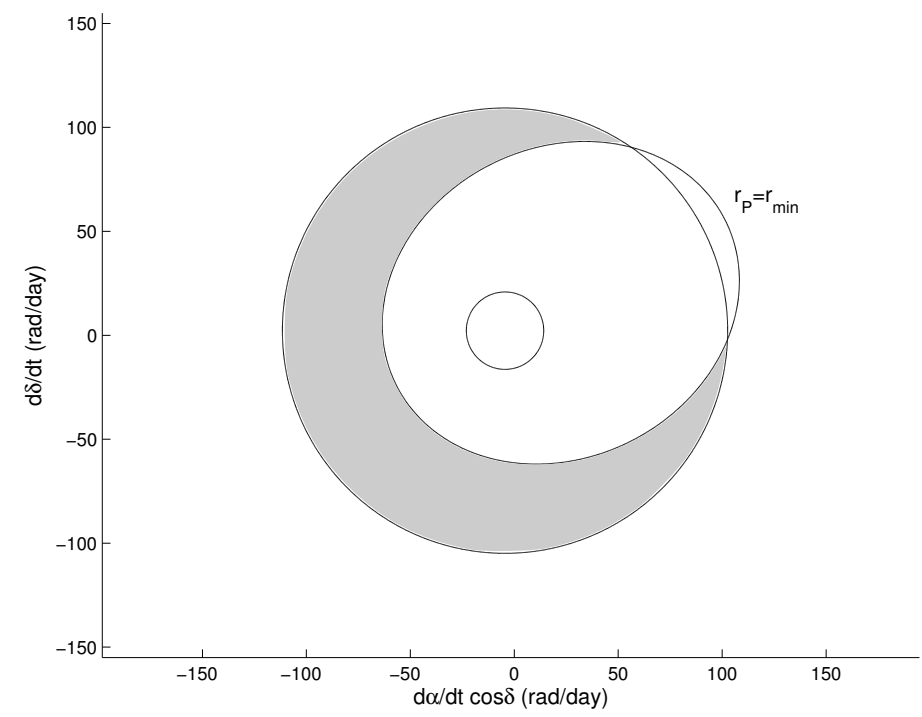

Figure 4: An example of admissible region, with the further condition on the pericenter distance $\left(r_{P} \geq r_{\min }\right)$, bounded by an ellipse.

\subsection{Virtual debris algorithm}

The admissible region can be used to generate a swarm of virtual debris: we sample it using the Delaunay triangulation [Milani et al. 2004] for the optical case and the cobweb [Tommei et al. 2007] for the radar case, as shown in Fig. 6 and 7 . The condition on the pericenter is not used at this step, because we could lose some important geometrical properties: this condition is used as filter, the nodes with a low pericenter are discarded.

The idea is to generate a swarm of virtual debris $X_{i}$, corresponding to the nodes of the admissible region of one of the two attributables, let us say $\mathcal{A}_{1}$. Then we compute, from each of the $X_{i}$, a prediction $\mathcal{A}_{i}$ for the epoch $t_{2}$, each with its covariance matrix $\Gamma_{\mathcal{A}_{i}}$. Thus for each virtual debris $X_{i}$ we can compute an attribution penalty $K_{4}^{i}$ [Milani et al. 2005], [Milani and Gronchi 2009][Cap. $8]$ and use the values as a criterion to select some of the virtual debris to proceed to the orbit computation.

Thus the procedure is as follows: we select some maximum value $K_{\max }$ for the attribution penalty and if there are some nodes such that $K_{4}^{i} \leq K_{\max }$ we 


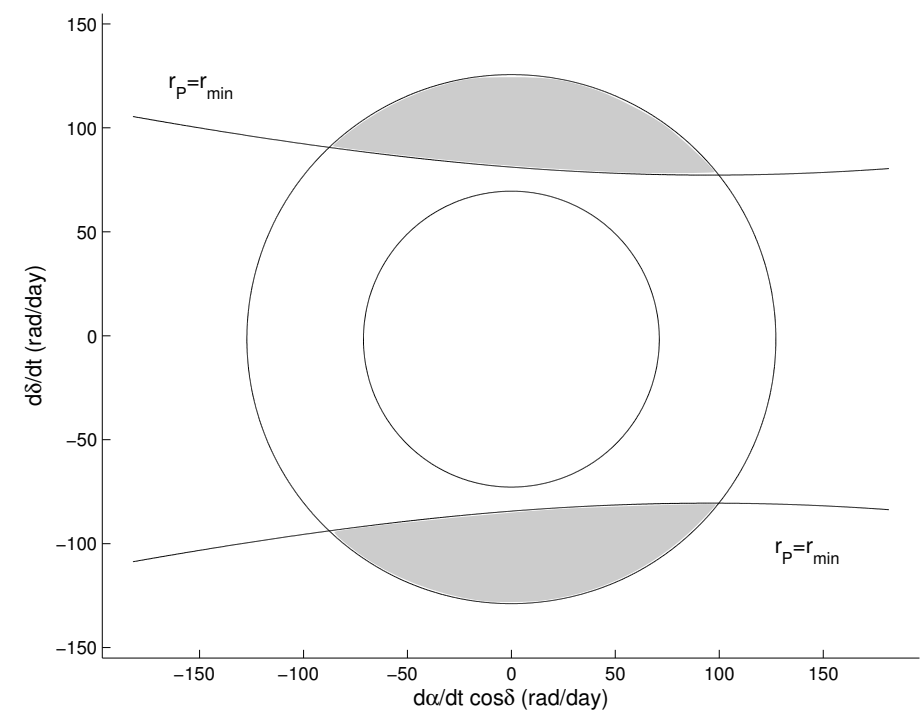

Figure 5: An example of admissible region, with the further condition on the pericenter distance $\left(r_{P} \geq r_{m i n}\right)$, bounded by a hyperbola.

proceed to the correlation confirmation. If this is not the case, we can try with another method, such as the one described in Sec. 4.

Note that other methods have been in use for long time to select a very small number of virtual debris; as an example, it is possible to select just circular orbits. [Fujimoto et al. 2010] show that is always possible to find from one to three circular orbits compatible with an optical attributable. Also this method can be useful if there is no confusion between the concept of virtual debris and the one of an orbit determined by the observations.

\subsection{Universal classification of objects}

The method of the admissible region is also useful to provide insight on the relationship between the different populations, in particular how they can mix in the observations. For a given optical attributable, supposedly computed from a short arc of optical observations, the Fig. 8 shows the region in the $(\rho, \dot{\rho})$ half-plane $\rho>0$ where Earth satellites (ES) can be, but also where ballistic trajectories (either launches L or reentries $\mathrm{R}$ ) can be, and where an asteroid serendipitously found in the same observations would be. Other more exotic populations, which are very unlikely, also have their region in the half plane: e.g., there are regions for direct departure/arrival to the Earth from interstellar space, which we have labeled as ET trajectories.

The same "universal" figure can be generated from a given radar attributable (Fig 9). In this case the regions corresponding to different populations partition 


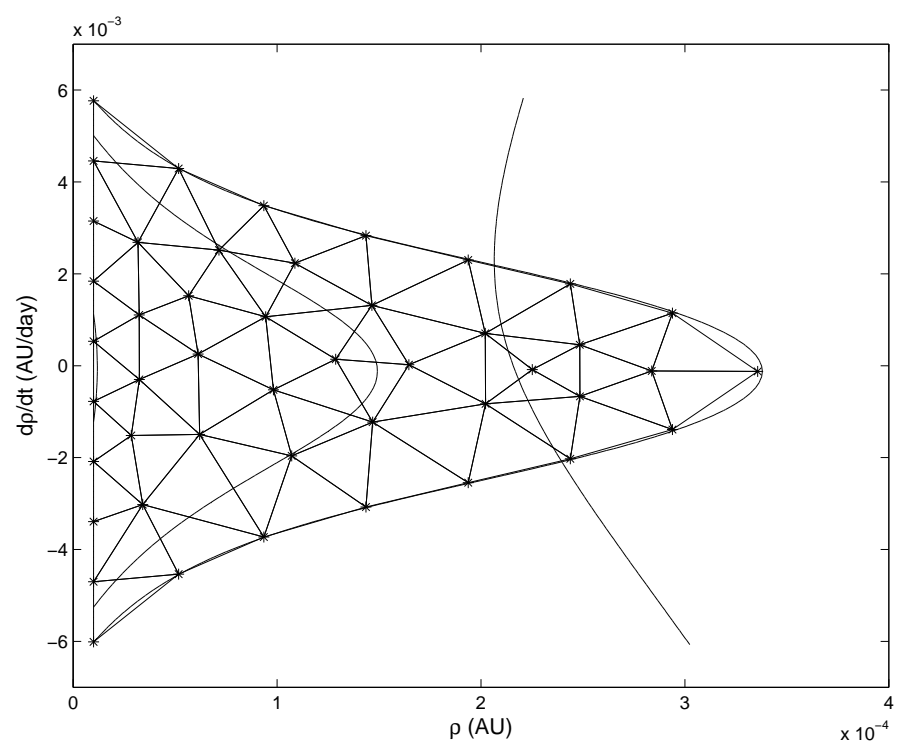

Figure 6: An example of admissible region, defined by imposing negative geocentric energy, for an optical attributable, with the Delaunay triangulation. The nodes of the triangulation corresponding to the ballistic trajectories (on the left of the curve cutting the outer part of the triangulation) can be discarded.

the plane $(\dot{\alpha} \cos \delta, \dot{\delta})$. The curve $\mathcal{E}_{\text {sun }}=0$, for the heliocentric energy, has been computed with formulas very similar to the ones for the geocentric energy.

\section{Keplerian integrals method}

We shall describe a method proposed for the asteroid case in [Gronchi et al. 2009] and based on the two-body integrals, to produce preliminary orbits starting from two attributables $\mathcal{A}_{1}, \mathcal{A}_{2}$ of the same object at two epoch times, $t_{1}$ and $t_{2}$. We assume that the orbit between $t_{1}$ and $t_{2}$ is well approximated by a Keplerian 2-body orbit, with constant energy $\mathcal{E}$ and angular momentum vector $\mathbf{c}$ :

$$
\left\{\begin{array}{l}
\mathcal{E}\left(t_{1}\right)-\mathcal{E}\left(t_{2}\right)=0 \\
\mathbf{c}\left(t_{1}\right)-\mathbf{c}\left(t_{2}\right)=0
\end{array} .\right.
$$

\subsection{Optical case}

Using (8), the second equation of (15) becomes

$$
\mathbf{D}_{1} \dot{\rho}_{1}+\mathbf{E}_{1} \rho_{1}^{2}+\mathbf{F}_{1} \rho_{1}+\mathbf{G}_{1}=\mathbf{D}_{2} \dot{\rho}_{2}+\mathbf{E}_{2} \rho_{2}^{2}+\mathbf{F}_{2} \rho_{2}+\mathbf{G}_{2} .
$$




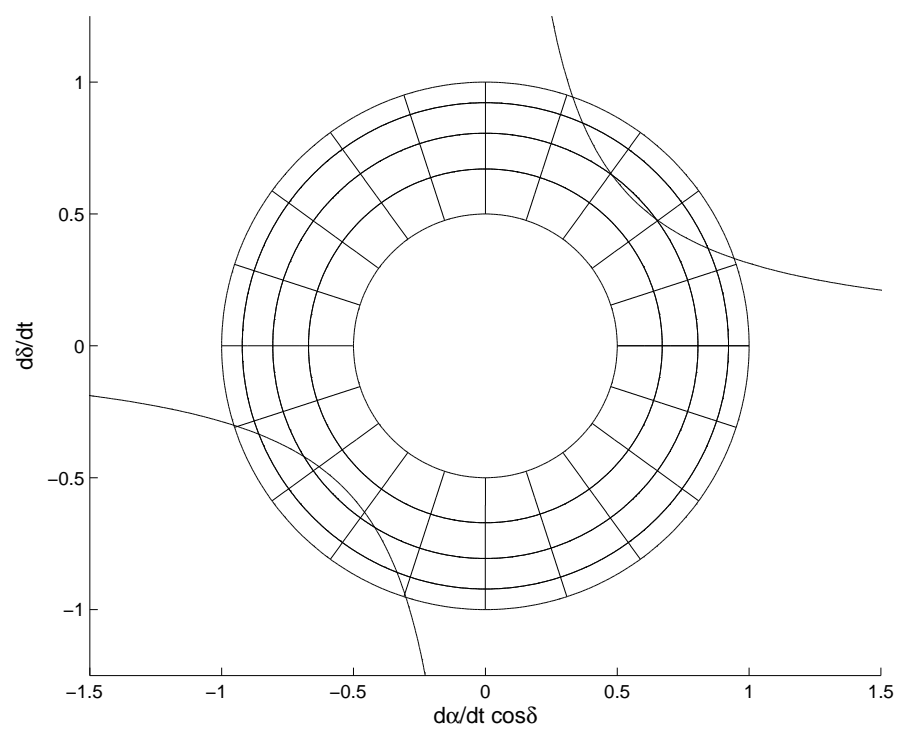

Figure 7: An example of admissible region, defined by imposing $\mathcal{E}_{\text {min }} \leq \mathcal{E} \leq 0$, for a radar attributable, with the cobweb sampling. The nodes of the cobweb corresponding to the ballistic trajectories (between the two branches of the hyperbola) can be discarded.

By scalar product between (16) and $\mathbf{D}_{1} \times \mathbf{D}_{2}$ we obtain the equation of degree 2 :

$$
q\left(\rho_{1}, \rho_{2}\right)=q_{11} \rho_{1}^{2}+q_{22} \rho_{2}^{2}+2 q_{13} \rho_{1}+2 q_{23} \rho_{2}+q_{33}=0,
$$

$q_{11}=\left(\mathbf{D}_{1} \times \mathbf{D}_{2}\right) \cdot \mathbf{E}_{1}, \quad q_{13}=\left(\mathbf{D}_{1} \times \mathbf{D}_{2}\right) \cdot \mathbf{F}_{1} / 2, \quad q_{33}=\left(\mathbf{D}_{1} \times \mathbf{D}_{2}\right) \cdot\left(\mathbf{G}_{1}-\mathbf{G}_{2}\right)$,

$q_{22}=-\left(\mathbf{D}_{1} \times \mathbf{D}_{2}\right) \cdot \mathbf{E}_{2}, \quad q_{23}=-\left(\mathbf{D}_{1} \times \mathbf{D}_{2}\right) \cdot \mathbf{F}_{2} / 2$.

Geometrically, this equation defines a conic section in the $\left(\rho_{1}, \rho_{2}\right)$ plane. By vector product between (16) and $\mathbf{D}_{2}$ followed by scalar product with $\mathbf{D}_{1} \times \mathbf{D}_{2}$ we have

$$
\dot{\rho}_{1}=\frac{\left(\mathbf{E}_{2} \rho_{2}^{2}+\mathbf{F}_{2} \rho_{2}+\mathbf{G}_{2}-\mathbf{E}_{1} \rho_{1}^{2}-\mathbf{F}_{1} \rho_{1}-\mathbf{G}_{1}\right) \times \mathbf{D}_{2}}{\left\|\mathbf{D}_{1} \times \mathbf{D}_{2}\right\|^{2}} \cdot\left(\mathbf{D}_{1} \times \mathbf{D}_{2}\right) .
$$

In a similar way the expression for $\dot{\rho}_{2}$ is

$$
\dot{\rho}_{2}=\frac{\mathbf{D}_{1} \times\left(\mathbf{E}_{1} \rho_{1}^{2}+\mathbf{F}_{1} \rho_{1}+\mathbf{G}_{1}-\mathbf{E}_{2} \rho_{2}^{2}-\mathbf{F}_{2} \rho_{2}-\mathbf{G}_{2}\right)}{\left\|\mathbf{D}_{1} \times \mathbf{D}_{2}\right\|^{2}} \cdot\left(\mathbf{D}_{1} \times \mathbf{D}_{2}\right) .
$$

Using the above formulae the energies $\mathcal{E}_{1}, \mathcal{E}_{2}$ can be considered as functions of $\rho_{1}, \rho_{2}$ only. Thus we obtain:

$$
\left\{\begin{array}{l}
\mathcal{E}_{1}\left(\rho_{1}, \rho_{2}\right)-\mathcal{E}_{2}\left(\rho_{1}, \rho_{2}\right)=0 \\
q\left(\rho_{1}, \rho_{2}\right)=0
\end{array}\right.
$$




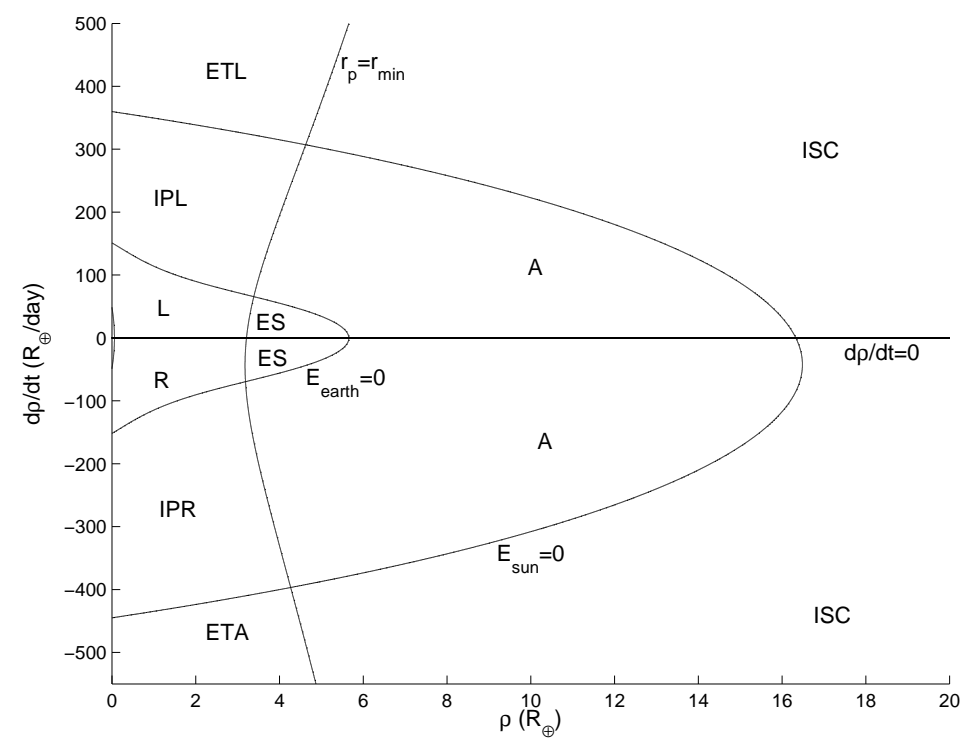

Figure 8: Partitioning of the $(\rho, \dot{\rho})$ half plane $\rho>0$ in regions corresponding to different populations, for an optical attributable with proper motion $\eta=10.1980$ $\mathrm{rad} /$ day. The labels mean: L Launch, R Reentry, ES Earth Satellite, A Asteroid, ISC Interstellar Comet, ETA ET Arriving, ETL ET Leaving, IPR Interplanetary Reentry, IPL Interplanetary Launch.

a system of 2 equations in 2 unknowns, already present in [Taff and Hall 1977]: they proposed a Newton-Raphson method to solve the system, but this results in a loss of control on the number of solutions. In [Gronchi et al. 2009] the authors have applied the same equations to the asteroid problem, and proposed a different approach to the solution of the system.

The energy equation is algebraic, not polynomial, because there are denominators containing square roots. By squaring twice it is possible to obtain a polynomial equation $p\left(\rho_{1}, \rho_{2}\right)=0$ of order 24 . Thus the system

$$
\left\{\begin{array}{l}
p\left(\rho_{1}, \rho_{2}\right)=0 \\
q\left(\rho_{1}, \rho_{2}\right)=0
\end{array}\right.
$$

has exactly 48 solutions in the complex domain, counting them with multiplicity. Of course we are interested only in solutions with $\rho_{1}, \rho_{2}$ real and positive; moreover, the squaring of the equations introduces spurious solutions. Nevertheless, we have found examples with up to 11 nonspurious solutions.

We need a global solution of the algebraic system of overall degree 48, providing all the possible couples $\left(\rho_{1}, \rho_{2}\right)$. This is a classical problem in algebraic geometry, which can be solved with the resultant method. We build an auxiliary $22 \times 22$ Sylvester matrix, having polynomials in $\rho_{2}$ as coefficients; its 


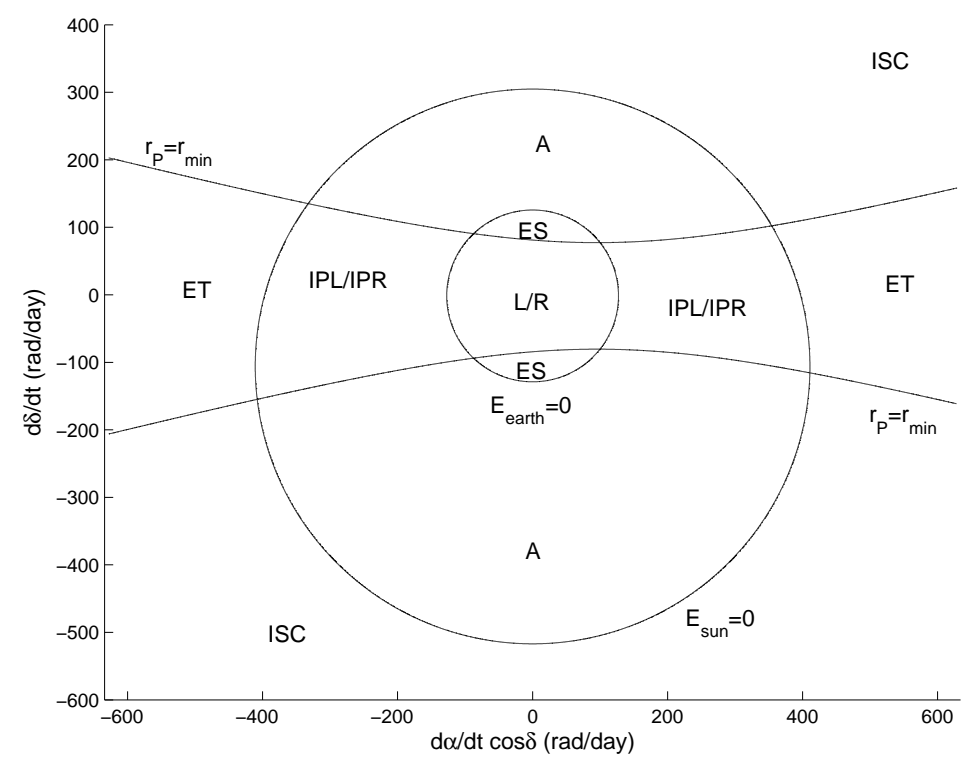

Figure 9: Partitioning of the $(\dot{\alpha} \cos \delta, \dot{\delta})$ plane in regions corresponding to different populations, for a radar attributable with $\rho=1 R_{\oplus}$. The labels mean: $\mathrm{L} / \mathrm{R}$ Launch or Reentry, ES Earth Satellite, A Asteroid, ISC Interstellar Comet, ET interstellar launch/reentry, IPL/IPR Interplanetary Launch or Reentry.

determinant, the resultant, is a polynomial of degree 48 in $\rho_{2}$ only. The values of $\rho_{2}$ appearing in the solutions of the polynomial system are the roots of the resultant [Cox et al. 1996].

Because the computation of the resultant is numerically unstable due to the wide range of orders of magnitude of the coefficients, one must use quadruple precision. Once the resultant is available, there are methods to solve the univariate polynomial equations, providing at once all the complex roots with rigorous error bounds [Bini 1996]. Given all the roots which could be real, we solve for the other variable $\rho_{1}$, select the positive couples $\left(\rho_{1}, \rho_{2}\right)$ and remove the spurious ones due to squaring. If the number of remaining solutions is zero, the attributables cannot be correlated with this method.

\subsection{Radar case}

The formulae for geocentric energy and angular momentum are given by (13) and (14), polynomials of degree 2 and 1 in the unknowns $(\dot{\alpha}, \dot{\delta})$, respectively. The system (15) has overall algebraic degree 2; such a system can be solved by elementary algebra.

The angular momentum equations are

$$
\mathbf{A}_{1} \dot{\alpha}_{1}+\mathbf{B}_{1} \dot{\delta}_{1}+\mathbf{C}_{1}=\mathbf{A}_{2} \dot{\alpha}_{2}+\mathbf{B}_{2} \dot{\delta}_{2}+\mathbf{C}_{2},
$$


which represents a system of 3 linear equations in 4 unknowns $\left(\dot{\alpha}_{1}, \dot{\delta}_{1}, \dot{\alpha}_{2}, \delta_{2}\right)$ and can be solved for three unknowns as a function of one of the four. For example, if we take the scalar product between (17) and $\mathbf{B}_{1} \times \mathbf{A}_{2}$ we have

$$
\dot{\alpha}_{1}=\frac{\mathbf{A}_{2} \cdot\left(\mathbf{B}_{1} \times \mathbf{B}_{2}\right) \dot{\delta}_{2}-\left(\mathbf{C}_{1}-\mathbf{C}_{2}\right) \cdot\left(\mathbf{A}_{2} \times \mathbf{B}_{1}\right)}{\mathbf{B}_{1} \cdot\left(\mathbf{A}_{1} \times \mathbf{A}_{2}\right)},
$$

and in a similar way we obtain

$$
\begin{gathered}
\dot{\delta}_{1}=\frac{\mathbf{B}_{2} \cdot\left(\mathbf{A}_{1} \times \mathbf{A}_{2}\right) \dot{\delta}_{2}-\left(\mathbf{C}_{1}-\mathbf{C}_{2}\right) \cdot\left(\mathbf{A}_{1} \times \mathbf{A}_{2}\right)}{\mathbf{B}_{1} \cdot\left(\mathbf{A}_{1} \times \mathbf{A}_{2}\right)}, \\
\dot{\alpha}_{2}=\frac{\mathbf{A}_{1} \cdot\left(\mathbf{B}_{1} \times \mathbf{B}_{2}\right) \dot{\delta}_{2}-\left(\mathbf{C}_{1}-\mathbf{C}_{2}\right) \cdot\left(\mathbf{A}_{1} \times \mathbf{B}_{1}\right)}{\mathbf{B}_{1} \cdot\left(\mathbf{A}_{1} \times \mathbf{A}_{2}\right)} .
\end{gathered}
$$

When the equations for $\left(\dot{\alpha}_{1}, \dot{\alpha}_{2}, \dot{\delta}_{1}\right)$ as a function of $\dot{\delta}_{2}$ are substituted in the equation for the energies $\mathcal{E}_{1}\left(\dot{\alpha}_{1}, \dot{\delta}_{1}\right)=\mathcal{E}_{2}\left(\dot{\alpha}_{2}, \dot{\delta}_{2}\right)$ we obtain a quadratic equation in $\dot{\delta}_{2}$, which can be solved by elementary algebra, giving at most two real solutions. Geometrically, equation (17) can be described by a straight line in a plane, e.g., in $\left(\dot{\alpha}_{2}, \dot{\delta}_{2}\right)$, where the energy equation defines a conic section.

\subsection{Singularities}

There are some cases in which the Keplerian integrals method can not be applied.

In the optical case we have to avoid the condition $\mathbf{D}_{1} \times \mathbf{D}_{2}=\left(\mathbf{q}_{1} \times \hat{\boldsymbol{\rho}}_{1}\right) \times$ $\left(\mathbf{q}_{2} \times \hat{\boldsymbol{\rho}}_{2}\right)=0$. This can happen when:

- $\mathbf{q}_{1}$ is parallel to $\hat{\boldsymbol{\rho}}_{1}$, i.e., the observation at time $t_{1}$ is done at the observer zenith;

- $\mathbf{q}_{2}$ is parallel to $\hat{\boldsymbol{\rho}}_{2}$, i.e., the observation at time $t_{2}$ is done at the observer zenith;

- $\mathbf{q}_{1}, \mathbf{q}_{2}, \hat{\boldsymbol{\rho}}_{1}$ and $\hat{\boldsymbol{\rho}}_{2}$ are coplanar. This case arises whenever a geostationary object is observed from the same station at the same hour of distinct nights.

As it is normal, the mathematical singularity is surrounded by a neighborhood in which the method is possible for zero error (both zero observational error and zero rounding off in the computation), but is not applicable in practice due to the limited numerical accuracy; e.g., this method fails even for non-geostationary, nearly geosynchronous orbits with hours of observations over different nights differing by only a few minutes each night.

In the radar case the procedure fails only if the four vectors $\mathbf{A}_{1}, \mathbf{A}_{2}, \mathbf{B}_{1}$ and $\mathbf{B}_{2}$ do not generate a linear space of dimension 3, i.e., when:

$$
\left\{\begin{array}{l}
\mathbf{A}_{i} \cdot\left(\mathbf{B}_{1} \times \mathbf{B}_{2}\right)=0 \\
\mathbf{B}_{i} \cdot\left(\mathbf{A}_{1} \times \mathbf{A}_{2}\right)=0
\end{array} \quad i=1,2 .\right.
$$


For $i=1$ we obtain

$$
\left\{\begin{array}{l}
\rho_{1}^{2} \rho_{2}\left[\hat{\boldsymbol{\rho}}_{\delta 2} \cdot\left(\mathbf{r}_{1} \times \mathbf{r}_{2}\right)\right]\left[\mathbf{r}_{1} \cdot\left(\hat{\boldsymbol{\rho}}_{\alpha 1} \times \hat{\boldsymbol{\rho}}_{\delta 1}\right)\right]=0 \\
\rho_{1}^{2} \rho_{2}\left[\hat{\boldsymbol{\rho}}_{\alpha 2} \cdot\left(\mathbf{r}_{1} \times \mathbf{r}_{2}\right)\right]\left[\mathbf{r}_{1} \cdot\left(\hat{\boldsymbol{\rho}}_{\delta 1} \times \hat{\boldsymbol{\rho}}_{\alpha 1}\right)\right]=0
\end{array}\right.
$$

and for $i=2$ the formulae are analogous. Thus there is singularity when:

- $\mathbf{r}_{1}$ is parallel to $\mathbf{r}_{2}$;

- $\mathbf{r}_{i} \cdot\left(\hat{\boldsymbol{\rho}}_{\delta i} \times \hat{\boldsymbol{\rho}}_{\alpha i}\right)=\cos \delta_{i}\left(\mathbf{q}_{i} \cdot \hat{\boldsymbol{\rho}}_{i}+\rho_{i}\right)=0$, but this can never happen, apart from coordinate singularities, because $\mathbf{q}_{i} \cdot \hat{\boldsymbol{\rho}}_{i} \geq 0$;

- $\mathbf{r}_{1} \cdot\left(\hat{\boldsymbol{\rho}}_{\delta 1} \times \hat{\boldsymbol{\rho}}_{\alpha i}\right)=0$ and $\mathbf{r}_{1} \cdot\left(\hat{\boldsymbol{\rho}}_{\delta 1} \times \hat{\boldsymbol{\rho}}_{\delta i}\right)=0$, i.e., $\hat{\boldsymbol{\rho}}_{\alpha i}$ and $\hat{\boldsymbol{\rho}}_{\delta i}$ for $i=1,2$ belong to the orbital plane.

\subsection{Preliminary orbits}

Once a solution of (15) is computed the values of attributable elements can be obtained for the epochs $\bar{t}_{1}$ and $\bar{t}_{2}$, and they can be converted into the usual Keplerian elements:

$$
\left(a_{j}, e_{j}, I_{j}, \Omega_{j}, \omega_{j}, \ell_{j}\right), j=1,2,
$$

where $\ell_{j}$ are the mean anomalies. The first four Keplerian elements $\left(a_{j}, e_{j}, I_{j}, \Omega_{j}\right)$ are functions of the 2-body energy and angular momentum vectors $\mathcal{E}_{j}, \mathbf{c}_{j}$, and are the same for $j=1,2$. Thus the result can be assembled in the 8 -dimensional vector:

$$
H=\left(V, \Phi_{1}, \Phi_{2}\right), \quad V=(a, e, I, \Omega), \Phi_{1}=\left(\omega_{1}, \ell_{1}\right), \Phi_{2}=\left(\omega_{2}, \ell_{2}\right) .
$$

There are compatibility conditions between $\Phi_{1}$ and $\Phi_{2}$ to be satisfied if the two attributables belong to the same object:

$$
\omega_{1}=\omega_{2}, \ell_{1}=\ell_{2}+n\left(\bar{t}_{1}-\bar{t}_{2}\right)
$$

where $n=n(a)$ is the mean motion. We cannot demand the exact equality in the formulae above, because of various error sources, including the uncertainty of the attributable, and the changes on the Keplerian integrals due to the perturbations with respect to the 2 -body model. Thus we need a metric to measure in an objective way the residuals in the compatibility conditions.

\subsection{Covariance propagation}

The two attributables $\mathcal{A}_{1}, \mathcal{A}_{2}$ have been computed from the observations by using a least squares fit to the individual observations, thus $4 \times 4$ covariance matrices $\Gamma_{\mathcal{A}_{1}}$ and $\Gamma_{\mathcal{A}_{2}}$ are available; they can be used to form the block diagonal $8 \times 8$ covariance matrix for both attributables $\Gamma_{\mathcal{A}}$. The Keplerian integral method allows to compute explicitly the vector $H$ of (18) and, by means of the implicit function theorem, its partial derivatives, thus it is possible by the 
standard covariance propagation formula [Milani and Gronchi 2009][Sec. 5.5] to compute also $\Gamma_{H}$, the covariance of $H$. With another transformation we can compute the average elements $\Phi_{0}=\left(\Phi_{1}+\Phi_{2}\right) / 2$ (as the best value for the angular elements at time $\bar{t}_{0}=\left(\bar{t}_{1}+\bar{t}_{2}\right) / 2$ ) and the discrepancy $\Delta \Phi$ in the compatibility conditions (19), and to propagate the covariance also to this 8 dimensional vector:

$$
\Gamma_{\mathcal{A}} \Longrightarrow \Gamma_{H} \Longrightarrow \Gamma_{V, \Phi_{0}, \Delta \Phi}
$$

The above argument is a generalization of the one in [Gronchi et al. 2009], where explicit computations are given for the optical attributables case.

In the $8 \times 8$ covariance matrix $\Gamma_{V, \Phi_{0}, \Delta \Phi}$, the lower right $2 \times 2$ block is the marginal covariance matrix of $\Delta \Phi$, from which we can compute the normal matrix and the $\chi^{2}$ :

$$
C_{\Delta \Phi}=\Gamma_{\Delta \Phi}^{-1}, \quad \chi_{\Delta \Phi}^{2}=\Delta \Phi \cdot C_{\Delta \Phi} \Delta \Phi
$$

which can be used as control, that is the discrepancy in the compatibility conditions is consistent with the observation error and the correlation between the two attributables is considered possible only if $\chi_{\Delta \Phi}^{2} \leq \chi_{\max }^{2}$.

The upper left $6 \times 6$ block is the covariance matrix of the preliminary orbit, that is of the orbital elements set $\left(V, \Phi_{0}\right)$ (at epoch $\left.\bar{t}_{0}\right)$. Although this preliminary orbit is just a 2-body solution, it has an uncertainty estimate, arising from the (supposedly known) statistical properties of the observational errors. This estimate neglects the influence of perturbations, such as the spherical harmonics of the Earth gravity field, the lunisolar differential attraction and the non-gravitational perturbations; nevertheless, if the time span $\bar{t}_{2}-\bar{t}_{1}$ is short, the covariance obtained above can be a useful approximation.

\subsection{Precession model}

We can generalize the method, including the effect due to the non-spherical shape of the Earth. The averaged equation for Delaunay's variables $\ell, \omega, \Omega$, $L=\sqrt{\mu a}, G=L \sqrt{1-e^{2}}$ and $Z=G \cos I$ are [Roy 2005][Sec. 10.4]:

$$
\left\{\begin{array}{l}
\overline{\dot{\ell}}=n-\frac{3}{4} n\left(\frac{R_{\oplus}}{a}\right)^{2} \frac{J_{2}\left(1-3 \cos ^{2} I\right)}{\left(1-e^{2}\right)^{3 / 2}} \\
\overline{\dot{\omega}}=\frac{3}{4} n\left(\frac{R_{\oplus}}{a}\right)^{2} \frac{J_{2}\left(4-5 \sin ^{2} I\right)}{\left(1-e^{2}\right)^{2}} \\
\overline{\dot{\Omega}}=-\frac{3}{2} n\left(\frac{R_{\oplus}}{a}\right)^{2} \frac{J_{2} \cos I}{\left(1-e^{2}\right)^{2}} \\
\overline{\dot{L}}=\overline{\dot{G}}=\overline{\dot{Z}}=0
\end{array}\right.
$$

where $J_{2}$ is the coefficient of the second zonal spherical harmonic of the Earth gravity field. In applying the Keplerian integral method to the case with $J_{2}$ perturbation, one can no longer use conservation of angular momentum. Since 
$L=\sqrt{-\mu^{2} / 2 \mathcal{E}}, G=\|c\|$ and $Z=\mathbf{c} \cdot \hat{\mathbf{z}}$, we can replace (15) with:

$$
\left\{\begin{array}{l}
\mathcal{E}_{1}=\mathcal{E}_{2} \\
\mathbf{c}_{1} \cdot \hat{\mathbf{z}}=\mathbf{c}_{2} \cdot \hat{\mathbf{z}} \\
\left\|\mathbf{c}_{1}\right\|^{2}=\left\|\mathbf{c}_{2}\right\|^{2} \\
\cos \left(\Omega_{2}\right)=\cos \left(\Omega_{1}+\overline{\dot{\Omega}}\left(\bar{t}_{2}-\bar{t}_{1}\right)\right)
\end{array}\right.
$$

In the optical case the first equation is algebraic and by squaring twice is possible to obtain a polynomial equation; in the radar case this relation is already polynomial. The second and the third equations are always polynomial, while the last equation needs to be linearized in the parameter $\dot{\bar{\Omega}}\left(\bar{t}_{2}-\bar{t}_{1}\right)$ :

$$
\cos \Omega_{2}=\cos \Omega_{1}-\overline{\dot{\Omega}}\left(\bar{t}_{2}-\bar{t}_{1}\right) \sin \Omega_{1} .
$$

The following relationships hold [Roy 2005][Sec. 4.11]:

$$
\begin{aligned}
& \cos \Omega_{i}=\frac{\hat{\mathbf{z}} \times \mathbf{c}_{i}}{\left\|\hat{\mathbf{z}} \times \mathbf{c}_{i}\right\|} \cdot \hat{\mathbf{x}}, \quad \sin \Omega_{i}=\frac{\hat{\mathbf{z}} \times \mathbf{c}_{i}}{\left\|\hat{\mathbf{z}} \times \mathbf{c}_{i}\right\|} \cdot \hat{\mathbf{y}}, \quad a\left(1-e^{2}\right)=\frac{\left\|\mathbf{c}_{1}\right\|^{2}}{\mu}, \\
& \cos I=\frac{\mathbf{c}_{1} \cdot \hat{\mathbf{z}}}{\left\|\mathbf{c}_{1}\right\|}, \quad n=\sqrt{-\frac{8 \mathcal{E}_{1}^{3}}{\mu^{2}}}, \quad \dot{\dot{\Omega}}\left(t_{2}-t_{1}\right)=\frac{\xi \sqrt{-8 \mathcal{E}_{1}^{3}}\left(\mathbf{c}_{1} \cdot \hat{\mathbf{z}}\right)}{\left\|\mathbf{c}_{1}\right\|^{5}}
\end{aligned}
$$

where $\xi=-3 \mu J_{2} R_{\oplus}^{2}\left(\bar{t}_{2}-\bar{t}_{1}\right) / 2$. Substituting in (22) we obtain

$$
\frac{\hat{\mathbf{z}} \times \mathbf{c}_{2}}{\left\|\hat{\mathbf{z}} \times \mathbf{c}_{2}\right\|} \cdot \hat{\mathbf{x}}=\frac{\hat{\mathbf{z}} \times \mathbf{c}_{1}}{\left\|\hat{\mathbf{z}} \times \mathbf{c}_{1}\right\|} \cdot \hat{\mathbf{x}}-\frac{\xi \sqrt{-8 \mathcal{E}_{1}^{3}}\left(\mathbf{c}_{1} \cdot \hat{\mathbf{z}}\right)}{\left\|\mathbf{c}_{1}\right\|^{5}} \frac{\hat{\mathbf{z}} \times \mathbf{c}_{1}}{\left\|\hat{\mathbf{z}} \times \mathbf{c}_{1}\right\|} \cdot \hat{\mathbf{y}}
$$

Since $\|\hat{\mathbf{z}} \times \mathbf{c}\|=\|\mathbf{c}\| \sin I$ is constant we have:

$$
\left\|\mathbf{c}_{1}\right\|^{5}\left[\hat{\mathbf{z}} \times\left(\mathbf{c}_{2}-\mathbf{c}_{1}\right)\right] \cdot \hat{\mathbf{x}}=-\xi \sqrt{-8 \mathcal{E}_{1}^{3}}\left(\mathbf{c}_{1} \cdot \hat{\mathbf{z}}\right)\left(\hat{\mathbf{z}} \times \mathbf{c}_{1}\right) \cdot \hat{\mathbf{y}},
$$

that is an algebraic equation. Furthermore, by squaring twice in the optical case and only once in the radar case it is possible to obtain a polynomial equation.

Finally the new compatibility conditions, in place of (19) need to take into account the precession of the perigee and the secular perturbation in mean anomaly:

$$
\omega_{1}=\omega_{2}+\overline{\dot{\omega}}\left(\bar{t}_{1}-\bar{t}_{2}\right), \quad \ell_{1}=\ell_{2}+\overline{\dot{\ell}}\left(\bar{t}_{1}-\bar{t}_{2}\right) .
$$

The overall degree of system (21) is summarized in Table 1 . We conclude that this method is unpractical for optical attributables, could be used for radar attributables, with computational difficulties comparable with the optical case without precession.

To solve the problem (even in the optical case) we begin by considering the parametric problem $\overline{\dot{\Omega}}=K$, where $K$ is constant. Thus we replace (15) with:

$$
\left\{\begin{array}{l}
\mathcal{E}_{1}-\mathcal{E}_{2}=0 \\
R \mathbf{c}_{1}-R^{T} \mathbf{c}_{2}=0
\end{array}\right.
$$


Table 1: Degrees of the equations in system (21), in both optical and radar cases.

\begin{tabular}{lll}
\hline & Optical case & Radar case \\
\hline $\mathcal{E}_{1}=\mathcal{E}_{2}$ & 16 & 2 \\
$\mathbf{c}_{1} \cdot \hat{\mathbf{z}}=\mathbf{c}_{2} \cdot \hat{\mathbf{z}}$ & 2 & 1 \\
$\left\|\mathbf{c}_{1}\right\|^{2}=\left\|\mathbf{c}_{2}\right\|^{2}$ & 4 & 2 \\
$\cos \Omega_{2}=\cos \Omega_{1}-\dot{\dot{\Omega}}\left(\bar{t}_{2}-\bar{t}_{1}\right) \sin \Omega_{1}$ & 54 & 12 \\
Total & 6912 & 48 \\
\hline
\end{tabular}

where $R$ is the rotation by $\Delta \Omega / 2=K\left(\bar{t}_{2}-\bar{t}_{1}\right) / 2$ around $\hat{\mathbf{z}}$. This means that for a fixed value of $K$ the problem has the same algebraic structure of the unperturbed one. The only thing needed is to substitute $\mathbf{D}_{1}, \mathbf{E}_{1}, \mathbf{F}_{1}$ and $\mathbf{G}_{1}$ with $R \mathbf{D}_{1}, R \mathbf{E}_{1}, R \mathbf{F}_{1}$ and $R \mathbf{G}_{1}$ in the optical case and $\mathbf{A}_{1}, \mathbf{B}_{1}$ and $\mathbf{C}_{1}$ with $R \mathbf{A}_{1}, R \mathbf{B}_{1}$ and $R \mathbf{C}_{1}$ in the radar case; similarly the vectors with index 2 are multiplied by $R^{T}$.

The compatibility conditions contain the precession of the perigee and the secular perturbation in mean anomaly, related to the one of the node by linear equations

$$
\omega_{1}=\omega_{2}+K C_{\omega}\left(\bar{t}_{1}-\bar{t}_{2}\right), \quad \ell_{1}=\ell_{2}+\left(n+K C_{\ell}\right)\left(\bar{t}_{1}-\bar{t}_{2}\right),
$$

where the coefficients $C_{g}, C_{\ell}$ can be easily deduced from (20). Thus we can compute the $\chi_{\Delta \Phi}^{2}(K)$ and set up a simple procedure to minimize this by changing $K$, then the control on the acceptability of the preliminary orbit is

$$
\min _{K} \chi_{\Delta \Phi}^{2}(K) \leq \chi_{\max }^{2}
$$

\section{Correlation confirmation}

The multiple orbits obtained using the methods described in the previous sections are just preliminary orbits, solution of a 2-body approximation (as in the classical methods of Laplace and Gauss), or possibly of a $J_{2}$-only problem. They have to be replaced by least squares orbits, with a dynamical model including all the relevant perturbations.

Even after confirmation by least squares fit, it might still be the case that some linkages with just two attributables can be false, that is the two attributables might belong to different objects. This is confirmed by the tests with real data reported in [Tommei et al. 2009] for the virtual debris method and in [Milani et al. 2009] for the Keplerian integrals method. [Gronchi et al. 2009] have found the same phenomenon in a simulation of the application of the same algorithm to the asteroid case. Thus every linkage of two attributables needs to be confirmed by correlating a third attributable. 
The process of looking for a third attributable which can also be correlated to the other two is called attribution [Milani 1999, Milani et al. 2001]. From the available 2-attributable orbit with covariance we predict the attributable $\mathcal{A}_{P}$ at the time $t_{3}$ of the third attributable, and compare with $\mathcal{A}_{3}$ computed from the third set of observations. Both $\mathcal{A}_{P}$ and $\mathcal{A}_{3}$ come with a covariance matrix, we can compute the $\chi^{2}$ of the difference and use it as a test. For the attributions passing this test we proceed to the differential corrections. The procedure is recursive, that is we can use the 3 -attributable orbit to search for attribution of a fourth attributable, and so on. This generates a very large number of manyattributable orbits, but there are many duplications, corresponding to adding them in a different order.

By correlation management we mean a procedure to remove duplicates (e.g., $A=B=C$ and $A=C=B$ ) and inferior correlations (e.g., $A=B=C$ is superior to both $A=B$ and to $C=D$, thus both are removed). The output catalog after this process is called normalized. In the process, we may try to merge two correlations with some attributables in common, by computing a common orbit [Milani et al. 2005]. For a description of the sequence of steps in this procedure see [Milani and Knežević 2005].

\section{Conclusions}

We have described two algorithms to solve the linkage problem, that is to compute an orbit for an Earth-orbiting object observed in two well separated arcs. The first method exploits the geometric structure of the admissible region of negative geocentric energy orbits, which is sampled to generate virtual orbits. The latter are propagated in time to find other observations which could belong to the same object. The second method exploits the integrals of the 2-body problem, which are constant even over a significant time span and thus should apply to both observed arcs of the same object.

This top level description is enough to understand that the virtual debris algorithm should be applied to short time intervals between observed arcs, less than one orbital period or at most a few orbital periods. The Keplerian integrals method can be used for longer time spans, spanning several orbital periods; it is near to a singularity for very short time spans and in some other near-resonance conditions, such as observations of a geosynchronous orbits at the same hour in different nights. We conclude that each method should be used in the cases in which it is most suitable. Both algorithms have been tested for the optical case with real data from the ESA Optical Ground Station [Tommei et al. 2009, Milani et al. 2009] with good results. The analogous algorithms have been tested for asteroids in simulations of next generation surveys [Milani et al. 2005, Gronchi et al. 2009]. Future work should include the tests of the radar case and the solution of other related problem, like orbit identification between two objects for which an orbit is already available.

Acknowledgements Part of this work was performed in the framework of ESOC Contract No. 21280/07/D/CS, "Orbit Determination of Space Objects Based on Sparse 
Optical Data".

The authors wish to thank M. Granvik and J. M. Maruskin for their useful comments.

\section{References}

[Bini 1996] Bini, D. A.: Numerical Computation of Polynomial Zeros by Means of Aberth's Method. Numerical Algorithms. 13, 179-200 (1996).

[Cox et al. 1996] Cox, D. A., Little, J. B., O'Shea, D.: Ideals, Varieties and Algorithms. Springer (1996).

[Fujimoto et al. 2010] Fujimoto K., Maruskin J. D., Scheeres D. J.: Circular and zero-inclination solutions for optical observations of Earth-orbiting objects. Celestial Mechanics \& Dynamical Astronomy. 106, 157-182 (2010).

[Gronchi et al. 2009] Gronchi, G. F., Dimare, L., Milani, A.: Orbit Determination with the two-body Integrals. Celestial Mechanics \& Dynamical Astronomy. Accepted (2010).

[Maruskin and Scheeres 2009] Maruskin, J. M., Scheeres, D. J.: Metrics on the space of bounded Keplerian orbits and space situational awareness. Proc. of the 48th IEEE Conference and Decision Control. 5912-5917 (2009).

[Maruskin et al. 2009] Maruskin, J. M., Scheeres, D. J., Alfriend, K. T.: Correlation of Optical Observations of Objects in Earth Orbit. Journal of Guidance, Control, and Dynamics. 32, 194-209 (2009).

[Milani 1999] Milani, A.: The Asteroid Identification Problem I: recovery of lost asteroids. Icarus. 137, 269-292 (1999).

[Milani et al. 2001] Milani, A., Sansaturio, Chesley, S. R.: The Asteroid Identification Problem IV: Attributions. Icarus. 151, 150-159 (2001).

[Milani et al. 2004] Milani, A., Gronchi, G. F., de' Michieli Vitturi, M., Knežević, Z.: Orbit Determination with Very Short Arcs. I Admissible Regions. Celestial Mechanics \& Dynamical Astronomy. 90, 59-87 (2004).

[Milani et al. 2005] Milani, A., Gronchi, G. F., Knežević, Z., Sansaturio, M. E., Arratia, O.: Orbit Determination with Very Short Arcs. II Identifications. Icarus. 79, 350-374 (2005).

[Milani and Knežević 2005] Milani, A., Knežević, Z.: From Astrometry to Celestial Mechanics: Orbit Determination with Very Short Arcs. Celestial Mechanics \& Dynamical Astronomy. 92, 1-18 (2005).

[Milani and Gronchi 2009] Milani, A. , Gronchi, G.F.: Theory of orbit determination. Cambridge University Press (2009). 
[Milani et al. 2009] Milani, A., Gronchi, G. F., Farnocchia, D., Tommei, G., Dimare, L.: Optimization of space surveillance resources by innovative preliminary orbit methods. Proc. of the Fifth European Conference on Space Debris. 30 March-2 April 2009, Darmstadt, Germany, SP-672 on CD-Rom.

[Roy 2005] Roy, A. E.: Orbital Motion. Institute of Physics Publishing (2005).

[Taff and Hall 1977] Taff, L. G., Hall, D. L.: The use of angles and angular rates. I - Initial orbit determination. Celestial Mechanics \& Dynamical Astronomy. 16, 481-488 (1977).

[Tommei et al. 2007] Tommei, G., Milani, A., Rossi, A.: Orbit Determination of Space Debris: Admissible Regions. Celestial Mechanics \& Dynamical Astronomy. 97, pp. 289-304 (2007).

[Tommei et al. 2009] Tommei, G., Milani, A., Farnocchia, D., Rossi, A.: Correlation of space debris observations by the virtual debris algorithm. Proc. of the Fifth European Conference on Space Debris. 30 March-2 April 2009, Darmstadt, Germany, SP-672 on CD-Rom. 